\title{
Assessment of Tip Shape Effect on Rotor Aerodynamic Performance in Hover
}

\author{
Je Young Hwang* and Oh Joon Kwon** \\ Department of Aerospace Engineering, Korea Advanced Institute of Science and Technology, Daejeon 305-701, Korea
}

\begin{abstract}
In the present study, an unstructured mixed mesh flow solver was used to conduct a numerical prediction of the aerodynamic performance of the S-76 rotor in hover. For the present mixed mesh methodology, the near-body flow domain was modeled by using body-fitted prismatic/tetrahedral cells while Cartesian mesh cells were filled in the off-body region. A high-order accurate weighted essentially non-oscillatory (WENO) scheme was employed to better resolve the flow characteristics in the off-body flow region. An overset mesh technique was adopted to transfer the flow variables between the two different mesh regions, and computations were carried out for three different blade configurations including swept-taper, rectangular, and swept-taper-anhedral tip shapes. The results of the simulation were compared against experimental data, and the computations were also made to investigate the effect of the blade tip Mach number. The detailed flow characteristics were also examined, including the tip-vortex trajectory, vortex core size, and first-passing tip vortex position that depended on the tip shape.
\end{abstract}

Key words: Unstructured Mixed Meshes, Overset Mesh Technique, High-Order WENO Scheme, Sikorsky-76 Rotor Blade

\section{Introduction}

Hovering is a unique flight mode for helicopters that makes these vehicles useful for both civilian and military operations. The shape of the blade tip of a helicopter rotor is known to have a considerable impact on the overall performance of the rotor, blade airloads, noise, and tip-vortex trajectory. Experimental and computational studies have been carried out to improve the hover performance according to variations in the rotor tip shapes [1-6]. Of the various blade configurations that are possible, tapered, swept and anhedral tip shapes have been widely used for modern helicopters. A tapered tip improves the rotor efficiency, such as the Figure of Merit, since it reduces the outboard profile drag. However, this tip shape is prone to enter a premature stall, and also increases the risk of a high vibration in the cruising flight $[2,6]$. The swept tip blade reduces the tip Mach number, allowing the rotor to obtain a higher advance ratio and to operate at tip Mach numbers before the compressibility effect is manifested [7]. However, the sweep on the blade also has an effect in that it increases the torsional moment of the blade because the aerodynamic center shifts aft of the elastic blade axis [4]. The anhedral at the blade tip improves the hover performance of the rotor by increasing the vertical separation between the blade and the tip vortex. However, the anhedral tip also tends to increase the bending moments $[4,7]$. In this regard, it is difficult to design a rotor blade because trade offs are required when accounting for the merits and the demerits of each of the rotor tip shapes. In the conceptual stage of the rotor design, trade offs can be evaluated only by conducting experiments involving numerous test runs to change the tip shapes, which may be very expensive and inefficient.

Recently, state-of-the-art computational techniques and numerical methods have been extensively used to simulate the aerodynamic performance of advanced rotor blades [810]. However, an accurate prediction of the flow field around a rotorcraft still remains as one of the most challenging problems, even for modern computational fluid dynamics
This is an Open Access article distributed under the terms of the Creative Commons Attribution Non-Commercial License (http://creativecommons.org/licenses/by$\mathrm{nc} / 3.0 /$ which permits unrestricted non-commercial use, distribution, and reproduction in any medium, provided the original work is properly cited. (c) * M.S. Student

** Professor, Corresponding author: ojkwon@kaist.ac.kr 
(CFD). Unlike a fixed-wing aircraft, the tip vortex remains near the rotor blades, which affects the blade aerodynamic loading and also induces a highly impulsive noise. Therefore, one of the key issues in helicopter aerodynamics is to accurately capture the trajectory and to retain the strength of the trailing tip vortices.

In computational fluid dynamics, the flow field is numerically analyzed by dividing the physical space around an object into discrete regions. For the discretization of the flow domain, several mesh topologies have been developed, including structured, unstructured, and Cartesian meshes. Each of these mesh topologies has its own specific strengths and weaknesses. Structured mesh methods are good in resolving near-body features, such as a boundary layer. As a result of the regular index-based data structures, these methods can be easily extended to achieve a high-order spatial accuracy. However, modeling and mesh generation around complex geometries is quite difficult and timeconsuming. Unstructured meshes are well known to be more suitable to handle complex geometric configurations. However, the spatial accuracy is mostly limited to the second order. Cartesian mesh techniques exhibit some desirable features, including an easy mesh generation, local mesh refinement, simple data structures, and extension to a highorder spatial accuracy. However, the resolution of the shear flow at a high Reynolds number near the solid surface is quite challenging due to the non-body-fitted mesh characteristics [11].

High-order methods are commonly known to provide numerical solutions that are more accurate than low-order methods with a similar number of computational cells, and these are thus very practically useful for rotor aerodynamics by more accurately resolving the wake structure. In the framework of finite-volume methods, a high-order spatial accuracy is usually obtained by collecting information on the neighboring cells. In the case of the structured mesh approach, the neighbors can be easily constructed as a result of the use of coordinates designated by indices. Therefore, one-dimensional high-order reconstruction schemes, such as TVD (Total Variation Diminishing), ENO (Essentially NonOscillatory), WENO (Weighted ENO), and compact schemes can be easily realized [12-15]. For unstructured meshes, highorder polynomial data reconstruction can be adopted for high-order accurate, finite-volume methods [16]. However, a spatial accuracy higher than the second order is rarely used in practice mainly because excessive stencils are required to reconstruct multi-dimensional high-order polynomials on unstructured meshes, and this reconstruction results in a huge amount of computational overhead.

Dual mesh topologies have also been recently utilized to overcome the weakness of using a single mesh topology and to obtain a solution with a high-order accuracy. The OVERFLOW solver was developed by adopting a body-fitted structured grid in the near-body region and a structured Cartesian grid in the off-body domain [17]. The two grid zones are coupled with an overset mesh method, and OVERFLOW allows a spatial accuracy up to the sixth-order by using central differences and adopts mesh refinement based on a multi-block approach. The solver showed abilities in solving various rotor aerodynamic problems, but complex geometry modeling is still difficult and is time-consuming due to the nature of the structured grids. Recently, a hybrid mesh paradigm was used to develop the HELIOS (Helicopter Overset Simulations) flow solver in order to allow for more flexible modeling of complex geometries [18]. HELIOS employs a set of two existing codes: abody-fitted unstructured mesh NSU3D flow solver to model the near-body area and a serial high-order ARC3D flow solver on structured Cartesian grids for the off-body area. The two codes are coupled using a Python infrastructure [19-22]. The CHIMPS software is used to communicate the flow variables between the dualmesh systems. Flux computation is performed with secondorder accuracy in the near-body unstructured mesh region while a high-order accuracy is obtained up to the sixth order in the off-body Cartesian grid region. Although the flow solver was successfully used and various rotor aerodynamic problems were solved, the data interpolation between the two independent heterogeneous mesh solvers negatively impacts the solution efficiency and the parallel scalability of the coupled code. Also, the mesh adaptation at the off-body Cartesian grid region can be achieved only on a block basis due to the nature of the structured grids.

In the present study, an unstructured mixed mesh flow solver was developed to more efficiently and accurately simulate the flow around rotorcrafts. With this mixed mesh topology, body-fitted prismatic/tetrahedral cells were generated in the near-body flow domain while a Cartesianbased mesh is utilized in the off-body region. A secondorder node-based finite-volume method was adopted in the near-body domain while a cell-centered finite-volume method with a high-order spatial accuracy was applied in the outer domain. In order to achieve a high-order accuracy in the off-body Cartesian mesh region, the WENO scheme was adopted for the rotor wake to be more accurately and efficiently captured by overcoming the weakness of conventional, accurate second-order finite-volume methods on unstructured meshes. An unstructured mixed mesh flow solver was applied in order to assess the aerodynamic performance and to investigate the flow characteristics of the S-76 rotor during hover. The computations were made 
for three different blade tip shapes [23], including a swepttaper, rectangular, and swept-taper-anhedral types, and the results were compared with the experimental data that was available in terms of thrust, torque and figure of merit (FM) [24]. The flow characteristics were investigated according to the tip shape by examining the typical rotor flow features, such as the tip-vortex trajectory, vortex core size, and firstpassing tip vortex position. An application was also made to examine the effect of the Mach number of the blade tip on the rotor hover performance.

\section{Numerical Method}

The fluid motion was modeled with three-dimensional, compressible Reynolds-averaged Navier-Stokes equations. The equations can be written in an integral form for an arbitrary computational domain with a boundary as

$$
\iint_{\Omega} \frac{\partial}{\partial t} \vec{Q} d V+\oint_{\partial \Omega}(\vec{F}(Q) \cdot \vec{n}) d S=\oint_{\partial \Omega}(\vec{G}(Q) \cdot \vec{n}) d S+\iint_{\Omega} \vec{S}(Q) d V
$$

where $\vec{Q}$ is the vector of the primitive variables, $\vec{Q}=\left[\rho, \rho u, \rho v, \rho w, e_{0}\right]^{T}$, and $\vec{F}(Q)$ and $\vec{G}(Q)$ denote the convective and diffusive fluxes of these variables, respectively. $\vec{S}(Q)$ is the source term to account for the centrifugal acceleration of the rotating blade about the $\mathrm{z}$-axis. The governing equations are discretized using a vertex-centered finite-volume method in the near-field body-fitted mesh region to better resolve the viscous shear layer. In the off-body Cartesian mesh region, a cell-centered scheme was adopted to utilize the regularity and the orthogonality of the mesh. The convective flux term was evaluated by employing a flux-difference splitting scheme for Roe, whereas the diffusive flux term was computed by adopting a modified central-difference method. An implicit time integration algorithm based on a linearized second-order Euler backward differencing was used coupled with dual-time stepping to advance the solution in time. The linear system of equations is solved at each time step using a point Gauss-Seidel method. The Spalart-Allmaras one-equation turbulence model was adopted to solve the transport equation for the turbulent eddy viscosity. To better resolve the tip vortices, a rotationcurvature correction is included in the turbulence model equation [25].

\subsection{Near-Body Flow Solver}

For the near-body field region around the blades, a bodyfitted unstructured tetrahedral/prismatic mesh is adopted. This mesh offers great flexibility in treating complex geometries and also ensures a proper mesh resolution inside of the boundary layer near the solid wall with the packed prismatic elements. A vertex-centered scheme is adopted in this near-body region, and the flow domain is divided into a finite number of control volumes surrounding each vertex that are made of non-overlapping median-dual cells with boundary surfaces defined by the cell centroids and the mid-points of the edges of the cells. For the inviscid flux computation, a second-order spatial accuracy was achieved through a linear reconstruction procedure based on either the least-square method or the Green-Gauss technique. The slope limiter proposed by Venkatakrishnan [26] is applied to improve the stability across the steep gradient regions, such as the shock discontinuities.

\subsection{Off-Body Flow Solver}

The off-body region outside of the body-fitted mesh is discretized by adopting an unstructured cell-centered finitevolume method for Cartesian meshes. The volume mesh generation in this off-body region is conducted through a simple and robust cell division procedure. Starting with a coarse initial mesh or a single root cell, hexahedral elements were repeatedly subdivided to resolve the emerging features of the flow [27]. The flow computation in the off-body region was performed in an accurate high-order manner to better resolve the detailed flow features. The high-order accuracy could be achieved by adopting either a 3rd, 5th, or 7th-order WENO (Weighted Essentially Non-Oscillatory) scheme by using the weights of the sub-stencils based on the local smoothness of the numerical solution. This approach allows for a high-order accuracy to be achieved with a nonoscillatory approximation of the solution around the flow discontinuities [28-30]. In the present study, an octree-based data structure is adopted to provide easy and autonomous cell treatment. The information from the data structure enables the numerical stencils necessary for the 3rd, 5th, and 7 th-order spatial accuracy to be easily constructed.

When achieving a high-order spatial accuracy in the WENO scheme, more enlarged numerical stencils are required. In the present Cartesian meshes, cells with different sizes could be collected for the numerical stencils of the WENO reconstruction. Then the solution with a highorder accuracy may be polluted at the cell faces where the cell size changes abruptly. A virtual control volume was introduced in the present study to resolve this problem.

Figure 1 shows a typical example of the WENO scheme applied on the Cartesian mesh cells. When the flux between cells A and B is evaluated in an accurate high-order manner, virtual control volumes $\mathrm{C}$ and $\mathrm{D}$ with the same cell size are introduced for the WENO reconstruction. In a similar 


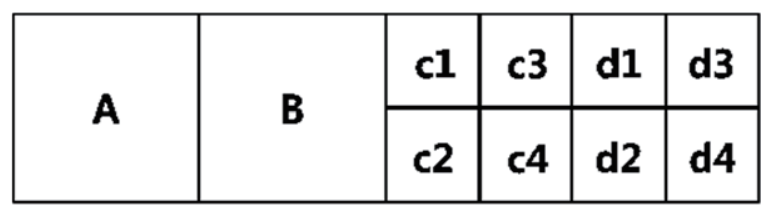

(a) Physical control volume

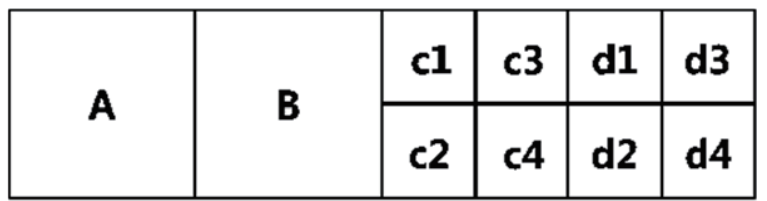

(b) Virtual control volume

Fig. 1. Construction of numerical stencils for the WENO scheme

manner, the flux between cells $\mathrm{cl}$ and $\mathrm{c} 3$ or between cells B and $\mathrm{c} 1$ is evaluated by introducing additional virtual control volumes b1 and b3. The flow information of the virtual control volumes is evaluated by interpolating them from the neighboring physical control volumes using a least-squares reconstruction.

\subsection{Overset Mesh Technique}

An overset mesh technique is used to exchange information of the flow variables between the near- and the off-body mesh blocks. For the overset mesh method, a search procedure is needed to identify the donor cells that contain vertices in the opposite overlapping mesh block. To overcome the large computational overhead involved in the search process, a fast and robust neighbor-to-neighbor $(\mathrm{N} 2 \mathrm{~N})$ search technique is implemented by taking advantage of the property of the linear shape functions. Once the search process has been completed, the information is used to clarify the node type and also to determine the weighting factors that are to be used for interpolation. In the present overset mesh method, a distance-to-wall technique was implemented to identify the active and non-active nodes. Hole-cutting is made to determine the cell types as either active, interpolation, non-active cells based on the number of active nodes attached to each cell. Then, the flow variables are interpolated between the two mesh blocks. This interpolation is performed with second-order accuracy using a linear shape function.

Figure 2 shows a typical example of the treatment of the special accuracy achieved across the block boundary for the off-body Cartesian mesh cells after hole-cutting. The cells on the left of the curved line in the figure represent those inside the near-body region and overlap with the tetrahedral/ prismatic cells. Here, the flow variables of the two Cartesian cell layers inside the boundary are interpolated with secondorder accuracy using those contained in the near-body mesh. This information is thus used to calculate the flow variables of the cells overlapping with the boundary line with third-order accuracy. Then, the accuracy of the computation on the cells outside the boundary gradually increases to a higher order as the neighboring numerical stencils are further enlarged.

\subsection{Parallel Computation}

A parallel computational algorithm based on a domain decomposition strategy is adopted to reduce the computational time required to handle a large number of cells. The global computational domain is partitioned into local subdomains using MeTiS libraries [31], and the domain decomposition is ordinarily performed for load balancing based on the computational cells. In the present study, virtual control volumes are constructed for highorder accuracy WENO reconstruction across the cells with a mismatching size after mesh adaptation, so the domain decomposition boundary may be set across the virtual control volumes. This problem can be avoided by dividing the local subdomains according to a tree-based partitioning method by considering both the physical and virtual control volumes.

Figure 3 shows the procedure for the tree-based partitioning method. At first, the computational mesh is constructed using the tree data structure, as in Fig. 3 (a). Then

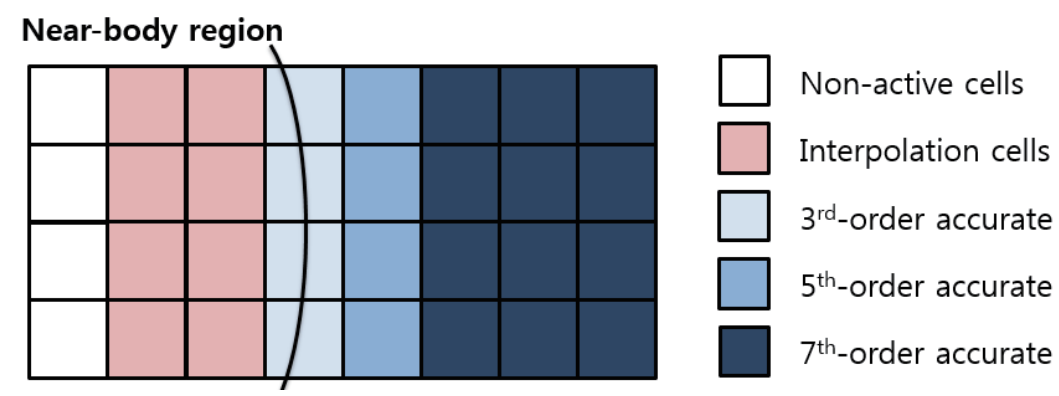

Fig. 2. Spatial order of the accuracy of the Cartesian mesh cells near the block boundary after hole-cutting 
the entire flow domain is divided into minimum depth trees, as shown in Fig. 3 (b), and the number of cells contained within each minimum depth tree is counted. Domain decomposition is performed for the minimum depth trees by balancing the total number of cells in each subdomain, as shown in Fig. 3 (c). While tree-based partitioning is used for the Cartesian meshes in the off-body region, cell-based partitioning is utilized for the unstructured mesh in the nearbody region. The Message Passing Interface (MPI) is then used to transfer the flow variables across the subdomain boundaries.

\section{Results and Discussion}

\subsection{S-76 Rotor in Hover}

An experimental S-76 rotor was simulated in hover to apply the present flow solver. The S-76 rotor consists of four blades and has a radius $(R)$ of 56.01 inches. Each blade has an aspect ratio of 18.077 and a chord length $(c)$ of 3.1 inches at a $75 \%$ span. Wind tunnel testing was conducted for three hovering tip Mach numbers $\left(\mathrm{M}_{\text {tip }}\right)$ of $0.55,0.60$ and 0.65 and for five blade configurations with different tip shapes [23]. The blade configurations that were considered included a rectangular planform and tips with a $20^{\circ}$ sweep, $60 \%$ taper, $35^{\circ}$ sweep with $60 \%$ taper, and $30^{\circ}$ sweep with $60 \%$ taper and $20^{\circ}$ anhedral. The experimental data, such as rotor thrust and the torque coefficients, were measured for several collective pitch angles $(\theta)$ ranging from 2 to 11 degrees [24].

In the present study, the effect of tip Mach number was examined by choosing the blade with a swept-tapered tip shape, and the computations were carried out with three tip Mach numbers of $0.55,0.60$, and 0.65 . In addition, the effect of the tip shape on the aerodynamic performance and the flow characteristics was verified by conducting simulations for three blade configurations with rectangular, swepttapered and swept-tapered-anhedral tip shapes. Figure 4 shows the schematic of the S-76 blade tip shapes and the computational modeling of the selected rotor blades. The computations for the tip Mach effect were performed for a collective pitch angle sweep from 6 to 11 degrees with a one degree interval. The pitch angle varied from 4 to 11 degrees during the test for the different tip shapes. In the computations, $3.5^{\circ}$ coning and a cylindrical center-body were considered to represent more realistic hovering flight conditions. All computations were conducted by using a 7th-order accurate WENO scheme for the off-body Cartesian mesh region. Convergence solutions were obtained with steady-state computations with 200 cores. The wall-clock

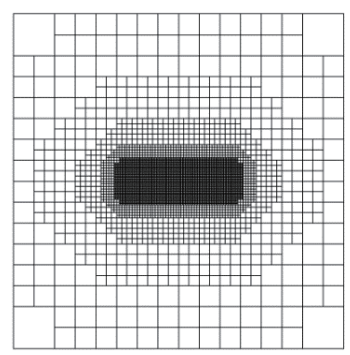

(a) Mesh generation

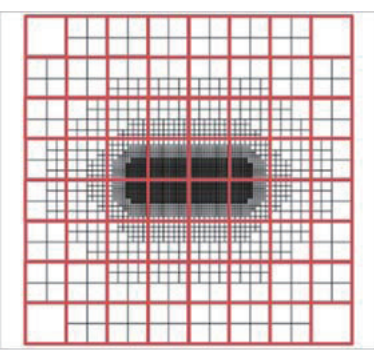

(b) Distribution into minimum depth trees

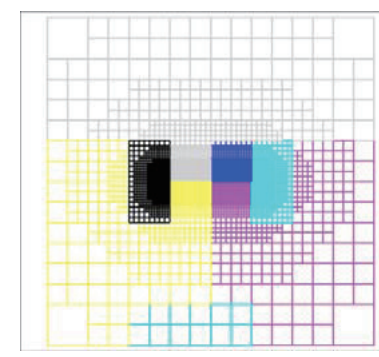

(c) Domain decomposition

Fig. 3. Domain decomposition strategy using the minimum depth tree

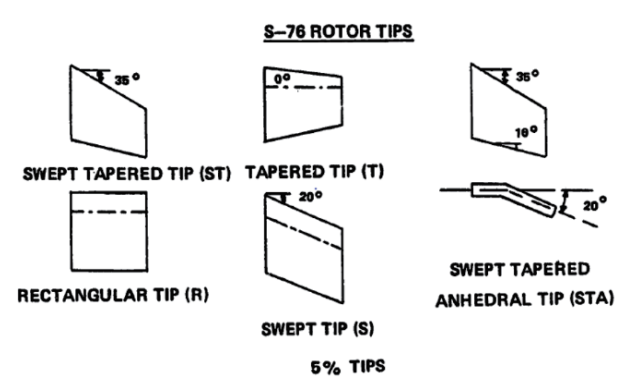

(a) Schematic of the S-76 blade tips

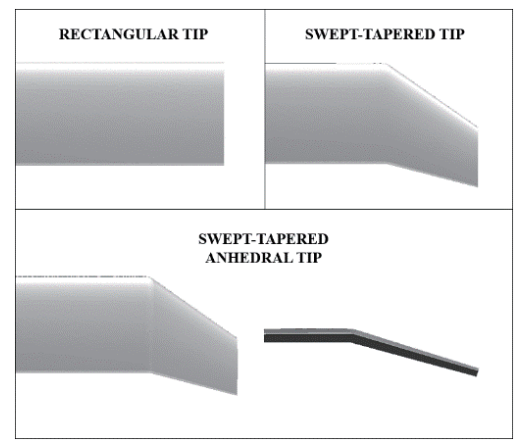

(b) Computational modeling of the S-76 blades

Fig. 4. S-76 blade tip geometries and computational geometry modeling 
time for a single case was of approximately 82 hours with 8,000 iterations. The thrust and torque coefficients were estimated with values averaged from the 7,000th iteration to the end.

\subsection{Computational Mesh}

Figure 5 presents the computational mesh for the S-76 blades. As can be seen in the figure, the near-body sub-block mesh around the blade and the center body is filled with prismatic/tetrahedral unstructured mesh cells. Relatively fine mesh cells are distributed in the blade tip region and along the trajectory of the initial tip vortex formation in order to more accurately capture the near-body flow features. The smallest cells in the near-body region have a typical size of a $1.5 \%$ chord length at the tip. Cartesian mesh cells are filled for the computational domain away from the blades and

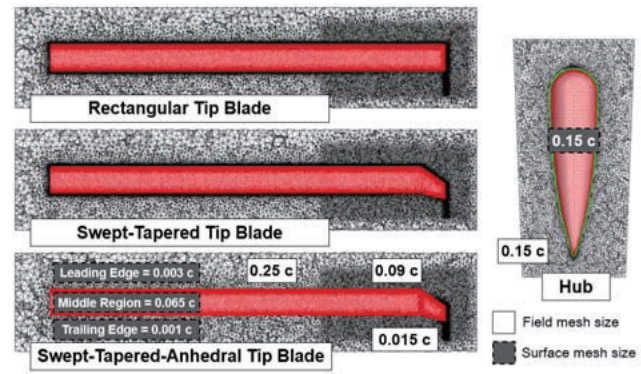

(a) Near-body mesh

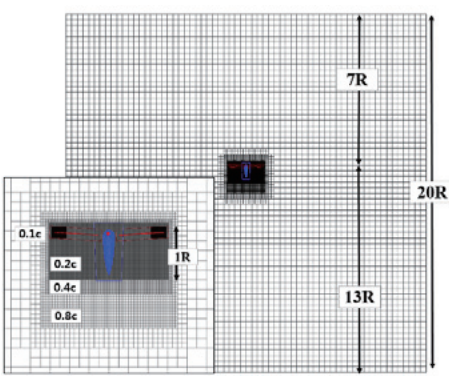

(b) Computation domain

Fig. 5. Computational mesh for the S-76 rotor simulations

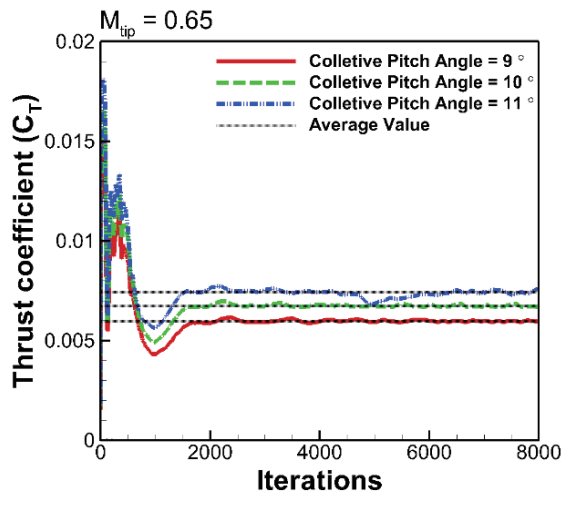

(a) Thrust coefficient vs iterations

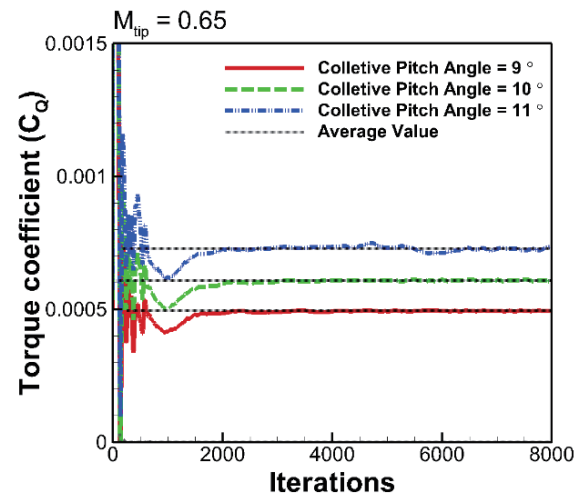

(b) Torque coefficient vs iterations

Fig. 6. Convergence history of the thrust and torque coefficient at a tip Mach number of 0.65 for collective pitch angle of $9^{\circ}, 10^{\circ}, 11^{\circ}$.

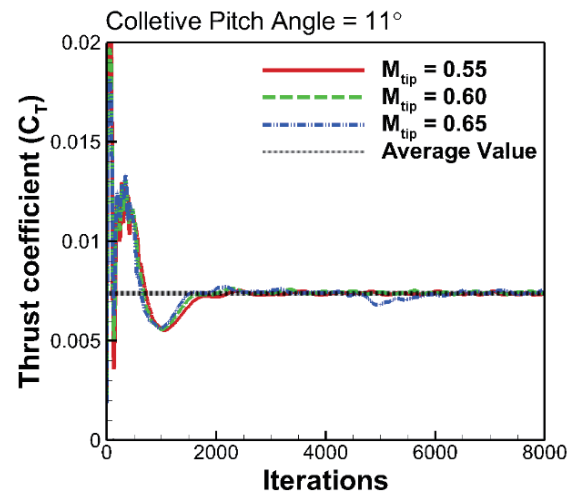

(a) Thrust coefficient vs iterations

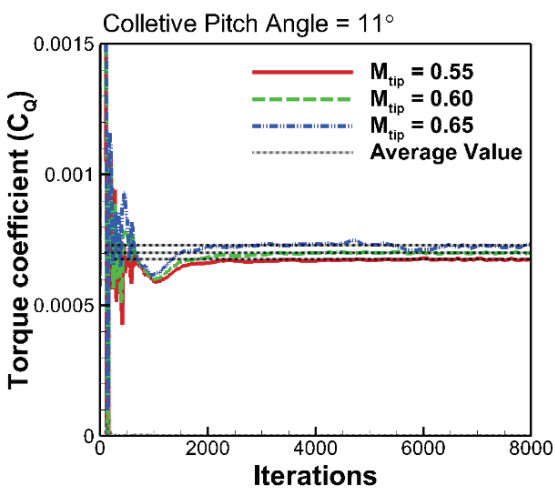

(b) Torque coefficient vs iterations

Fig. 7. Convergence history of thrust and torque coefficient for a collective pitch angle of $11^{\circ}$ at tip Mach numbers of $0.55,0.60,0.65$ 
the center body. The off-body mesh cells have a size with a $10 \%$ chord length in the near wake region. The complete computational domain has a size of 20 radii in all directions, and the upstream far-field boundary is located approximately 7 radii away from the rotor. The entire computational domain consists of approximately 7 million hexahedra in the offbody Cartesian mesh block, 4.3 million node points in each of the four blade sub-blocks and 0.2 million node points in the sub-block for the center-body. At the solid wall, the noslip boundary condition is treated with viscous flows, and the characteristic boundary condition is applied to the farfield boundary.

\subsection{Effect of the Tip Mach Number}

The effect of the blade tip Mach number was first examined with the blade with a swept-tapered tip shape that was chosen for this purpose. Computations were conducted for three tip Mach numbers of 0.55, 0.60, and 0.65, and the convergence histories of the thrust $\left(\mathrm{C}_{\mathrm{T}}\right)$ and torque coefficient $\left(C_{Q}\right)$ for high collective pitch angles are shown in Figs. 6 and

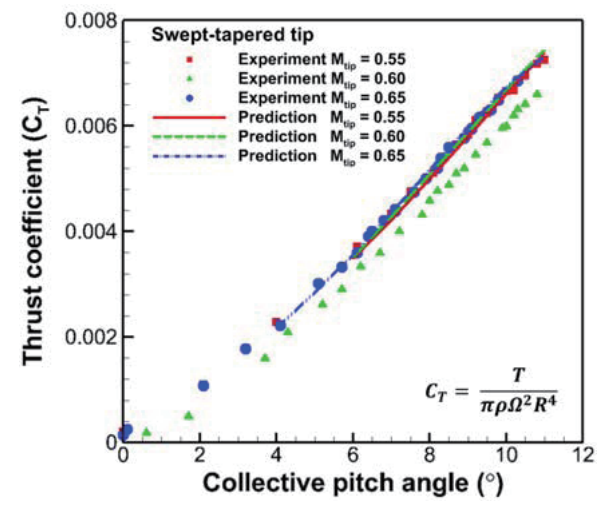

(a) Thrust coefficient vs collective pitch angle

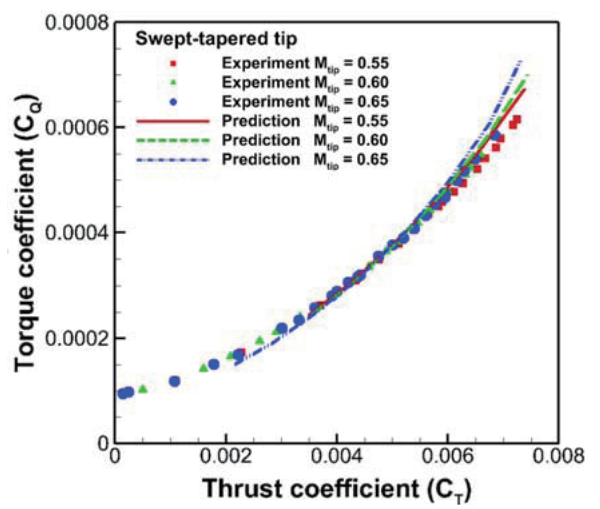

(c) Torque coefficient vs thrust coefficient
7. The predicted solutions are generally well converged, and the predicted thrust coefficient $\left(\mathrm{C}_{\mathrm{T}}\right)$, torque coefficient $\left(\mathrm{C}_{\mathrm{Q}}\right)$, and figure of merit (FM) are compared in Fig. 8 to those obtained from the experimental data [24]. The predicted thrust and torque variations are shown to be similar to each other for all three Mach numbers, except for the torque at relatively high collective pitch angles. The predicted results are in good agreement with the experiment for all blade collective pitch angles for two tip Mach numbers of 0.55 and 0.65 . However, for the case with a tip Mach number of 0.60 , the thrust coefficient obtained from the experiment is consistently lower by approximately $10 \%$ than that predicted at the same tip Mach number as well as relative to both the predicted and experimental values of the other two tip Mach numbers. The measured torque coefficient is also lower than that of the others by about $6 \%$ to $17 \%$. The overall behavior of the predicted torque vs. thrust is also consistent with that of the experiment, except at high collective pitch angles. As a result, the figure of merit is predicted to be slightly lower than that of the experiment, particularly at high pitch angles above 9 degrees.

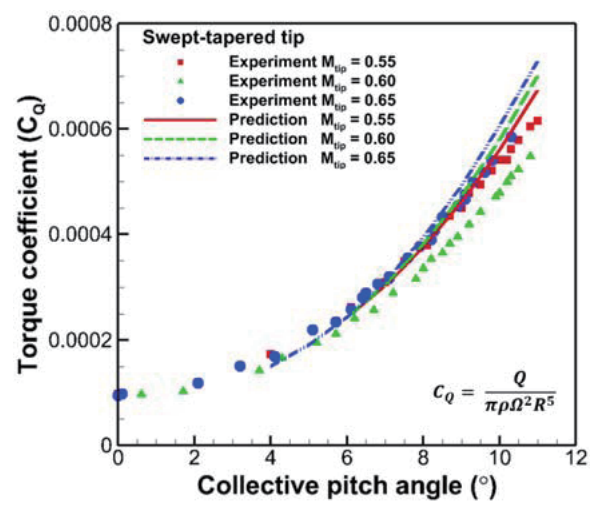

(b) Torque coefficient vs collective pitch angle

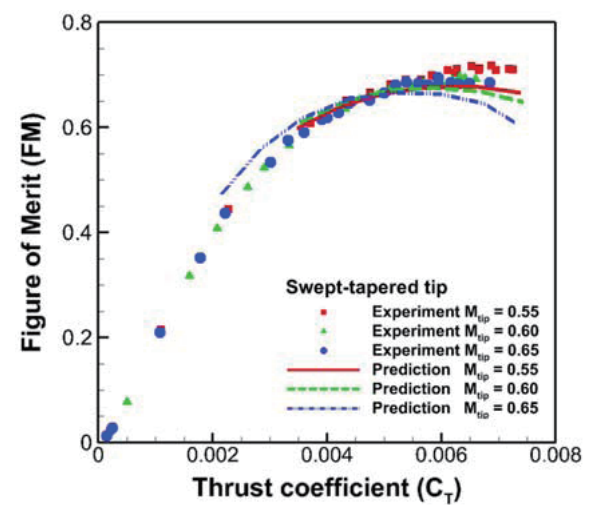

(d) Figure of merit vs thrust coefficient

Fig. 8. Comparison of the predicted and experimental aerodynamic performance parameters between different tip Mach numbers for the variation in the collective blade pitch angle 
In Fig. 9, the pressure coefficient distribution along the chord is compared at twelve selected radial stations ( $\mathrm{r} / \mathrm{R})$ between the different tip Mach numbers for a collective pitch angle of 11 degrees. The effect of the tip Mach number on the chordwise pressure distribution is shown to not be significant at inboard stations up to an $80 \%$ span. For further outboard stations, a slight deviation is observed between the different tip Mach numbers, mostly over the upper surface of the blade. At the $92.5 \%$ radial station, an abrupt change in the pressure can be observed over the front mid chord, which is an indication of the flow separation.

In Fig. 10, the pressure contours and the streamline patterns are compared to the upper surface of the rotor blade between the different tip Mach numbers at a collective pitch angle of 11 degrees. The flow is shown to be separate from the leading-edge of the swept tip, and leading-edge vortices form for all tip Mach numbers. As the tip Mach number increases, the size of separation bubble is also shown to gradually become larger, and the separated flow region is further expanded into the inboard blade stations. As a result of the increased flow separation, the thrust decreases and the torque correspondingly increases for the case of a tip Mach number of 0.65. This also results in a decrese in the figure of merit, as shown in Fig. 8.

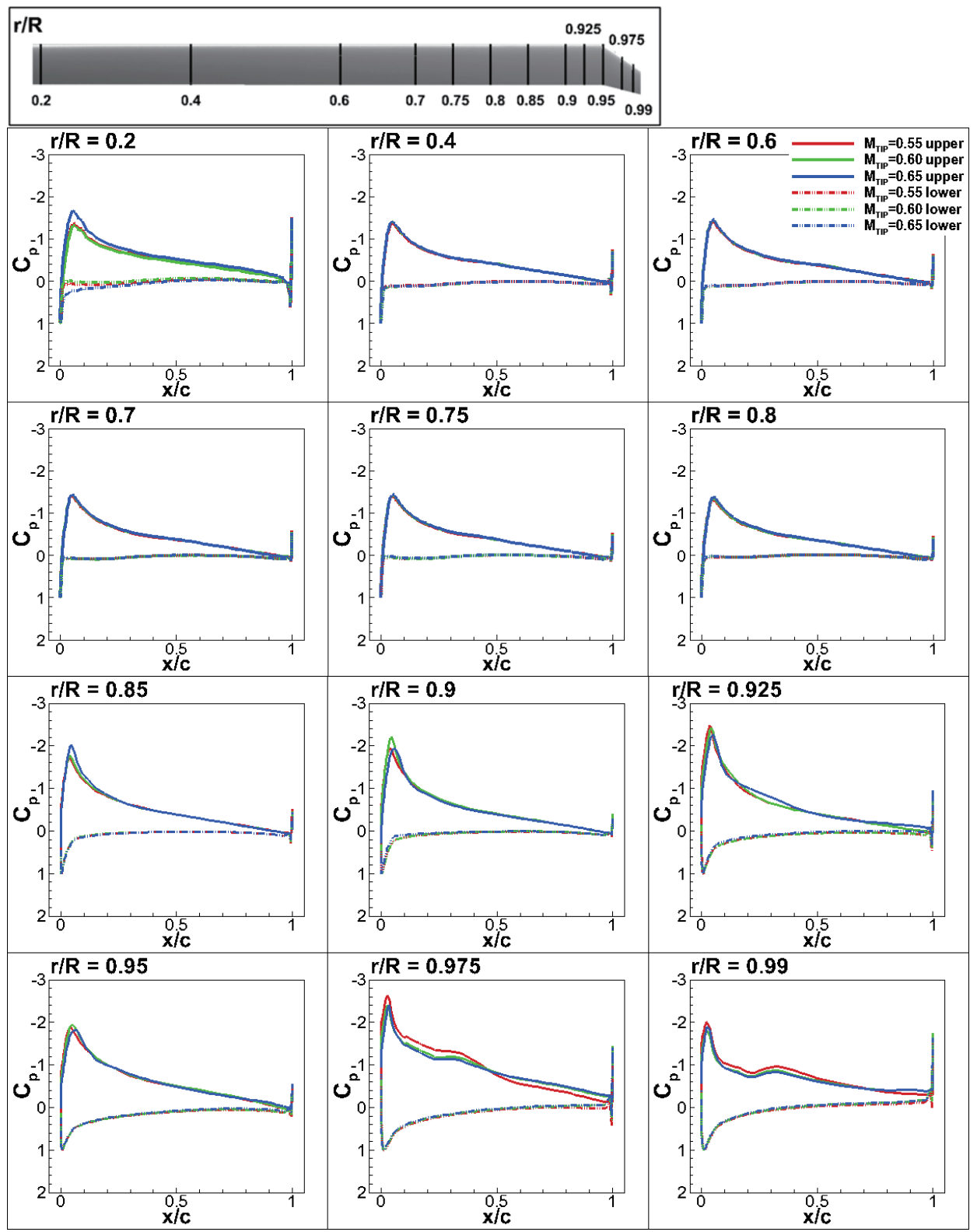

Fig. 9. Comparison of blade chordwise surface pressure distributions between different tip Mach numbers at twelve radial stations for the 11-degree collective pitch angle. 
In Fig. 11, the predicted tip-vortex trajectories from the rotor blade tip and the circulation strength of the tip vortex are presented in terms of the vortex age at an 11-degree collective pitch angle for different tip Mach numbers. The tip-vortex trajectories were obtained by connecting the vortex core centers at the cross-sections with an azimuthal increase of $5^{\circ}$. The vortex center was tracked by locating the center point between the low and high peaks of the sectional velocity at each azimuthal plane along the tip vortex. The circulation strength of the tip vortex was evaluated by integrating the distributed sectional vorticity inside of the vortex core. The circulation strength is presented in a nondimensional form of $\Gamma /\left(c_{\mathrm{e}} \Omega R\right)$, where ce represents the thrust weighted chord defined as $c_{e}=3 \int_{0}^{1} c(x) x^{2} d x$. The value of the thrust weighted chord for the swept-tapered blade is of 3.0278 , and the figure shows that the radial contraction of the wake increased slightly as the blade tip Mach number increased. In contrast, the vertical convection distances are similar to each other, regardless of the tip Mach number. As the tip Mach number increased, the tip vortex remains better resolved over a longer wake age. The strength of the tip- vortex circulation is also observed to become proportionally stronger as the tip Mach number increases.

\subsection{Effect of Blade Tip Shape}

Next, the effect that the tip shape had on the aerodynamic performance and flow characteristics was investigated. To this end, computations were carried out for three blade configurations with rectangular, swept-tapered and swepttapered-anhedral tips. A blade tip Mach number of 0.65 was chosen for these computations.

In Fig. 12, the predicted thrust coefficient $\left(\mathrm{C}_{\mathrm{T}}\right)$, torque coefficient $\left(\mathrm{C}_{\mathrm{Q}}\right)$, and figure of merit $(\mathrm{FM})$ for three different S-76 rotor blade configurations is compared with experimental data [24]. The overall behavior of the thrust and torque predicted for the three different blades was shown to be similar to those of the experiment. The three predicted values are similar at low pitch angles, but exhibit a slight deviation as the pitch angle increases. However, the predicted deviation that depends on the blade configuration is much smaller than that observed in the experiment. In general,

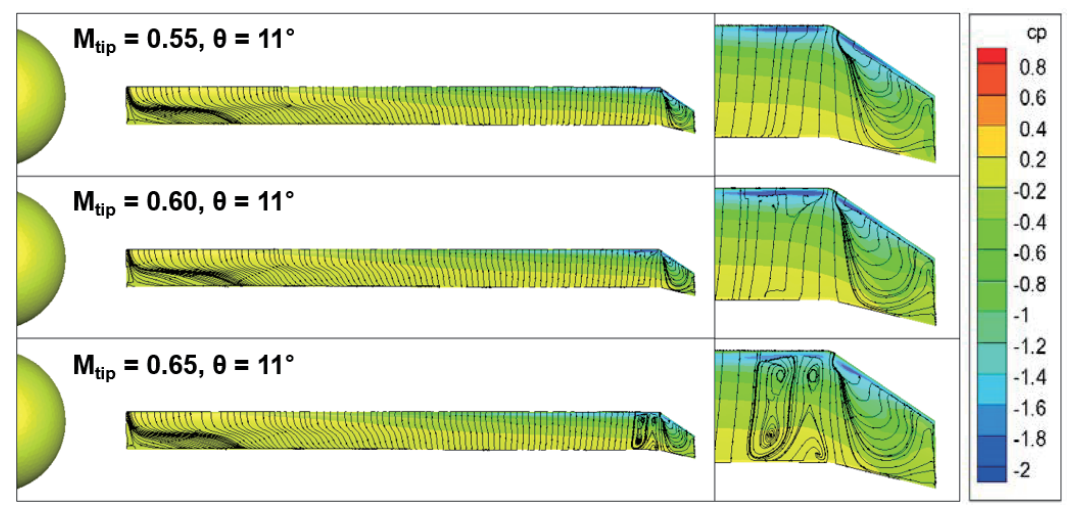

Fig. 10. Pressure coefficient contours and streamline patterns on the upper surface of the rotor blade for the 11-degree collective pitch angle

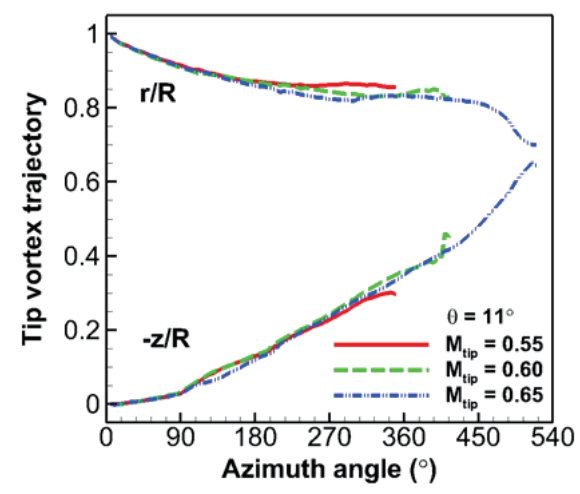

(a) Tip-vortex trajectory

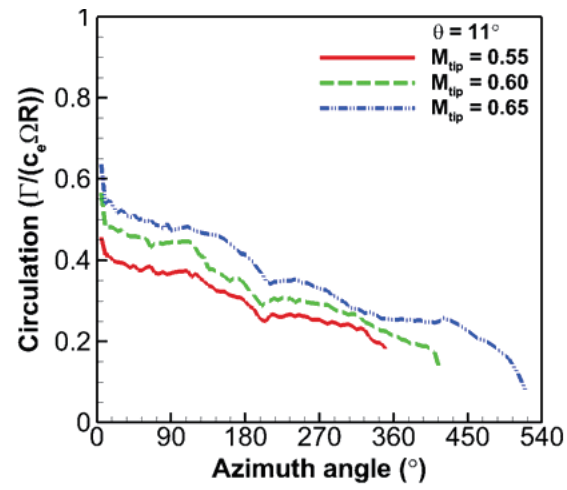

(b) Circulation in wake age

Fig. 11. Comparison of the trajectories and circulation strengths of tip vortex along the wake age between different tip Mach numbers for the 11-degree collective pitch angle 
the predicted thrust and torque of the rectangular tip are slightly higher than that of the other two tip configurations. Those of the swept-tapered-anhedral tip are the smallest of all, particularly for high collective pitch angles. In the case of the figure of merit, the anhedral tip shows the highest value, and the rectangular tip appears to have the lowest value. This tendency becomes more evident as the collective pitch angle increases further.

The collective pitch angle setting was estimated at a specified thrust value of $\mathrm{C}_{\mathrm{T}} / \sigma=0.09$ to examine the rotor trim for the three different blade configurations. The

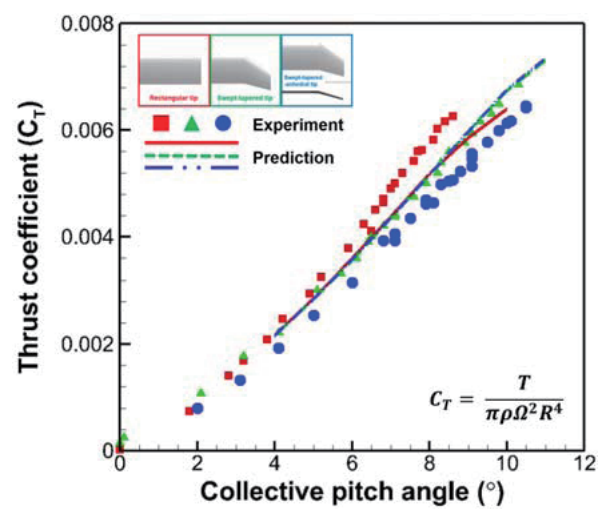

(a) Thrust coefficient vs collective pitch angle

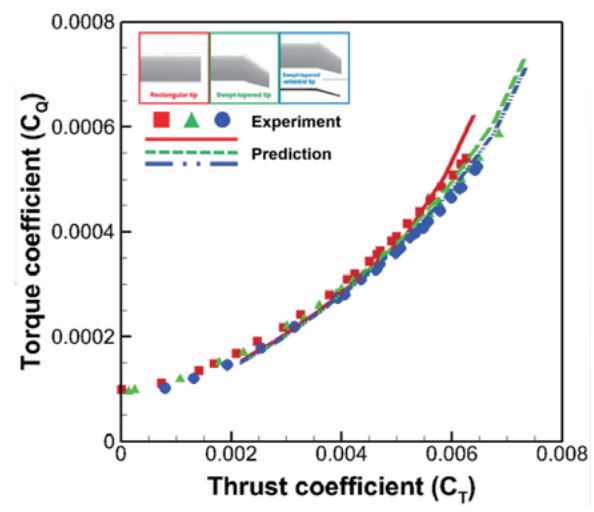

(c) Torque coefficient vs thrust coefficient computations were iteratively repeated until the error in the value of the predicted thrust becomes less than $0.1 \%$ of the specified one. The computation was initiated from a collective pitch angle estimated between 9 and 10 degrees for the specified thrust using the values predicted in Fig. 12. Then the collective pitch angle was updated by through a linear interpolating for the thrust change. The collective pitch angle setting corresponding to the specified thrust was obtained after four iterations, as shown in Fig. 13, and the convergence history of the thrust and the torque coefficient at the final states of the rotor trim are shown in Fig. 14. The

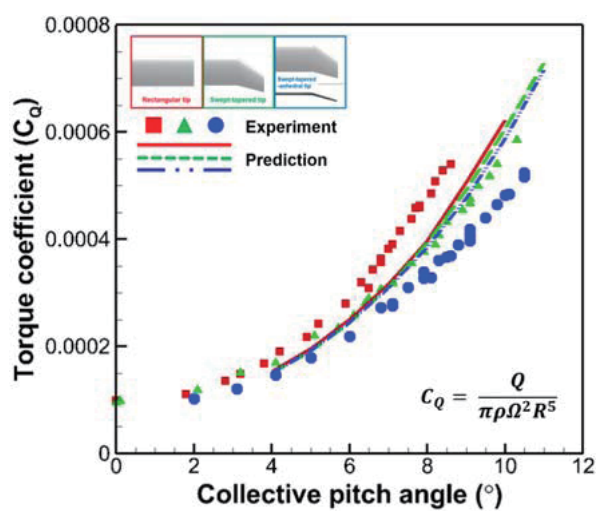

(b) Torque coefficient vs collective pitch angle

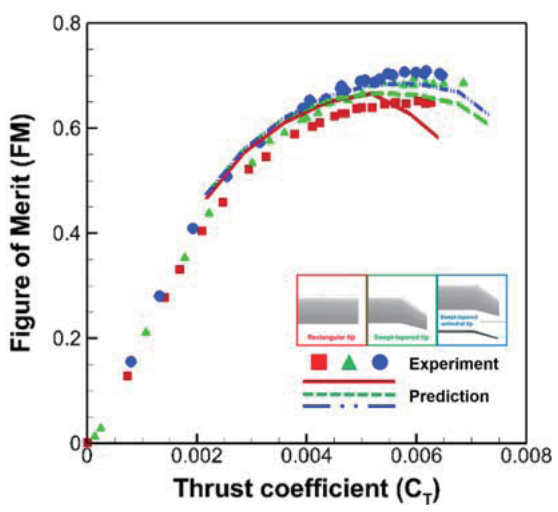

(d) Figure of merit vs thrust coefficient

Fig. 12. Comparison of predicted and experimental aerodynamic performance parameters for three different S-76 blade tip shapes
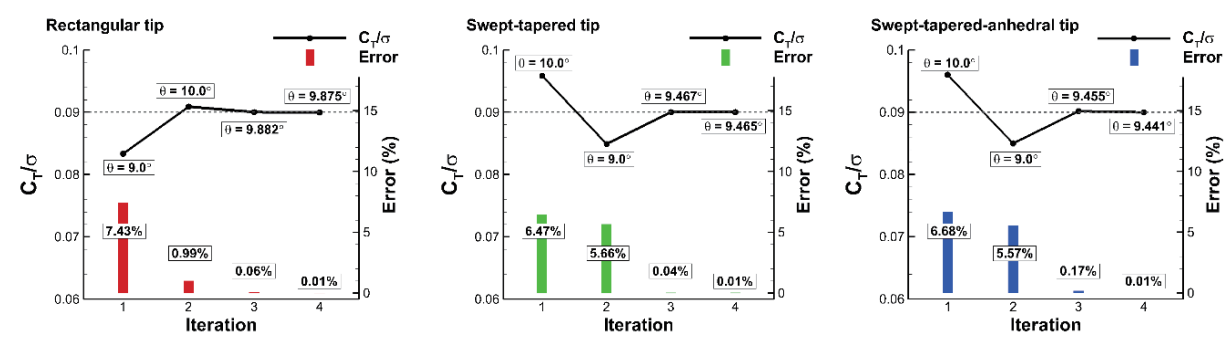

Fig. 13. Convergence history of the collective pitch angle for the specified thrust value of $\mathrm{CT} / \mathrm{\sigma}=0.09$ for different blade tip shapes at a 0.65 tip Mach number 
Table 1. Predicted aerodynamic performance parameters at the trimmed collective pitch angle setting

\begin{tabular}{ccccc}
\hline & $\theta$ & $C_{T} / \sigma$ & $C_{Q} / \sigma$ & FM \\
\hline Rectangular tip & $9.875^{\circ}$ & 0.089994 & 0.0086660 & 0.58504 \\
Swept-tapered tip & $9.465^{\circ}$ & 0.090013 & 0.0077343 & 0.65525 \\
Swept-tapered-anhedral tip & $9.441^{\circ}$ & 0.089991 & 0.0074454 & 0.68043 \\
\hline
\end{tabular}

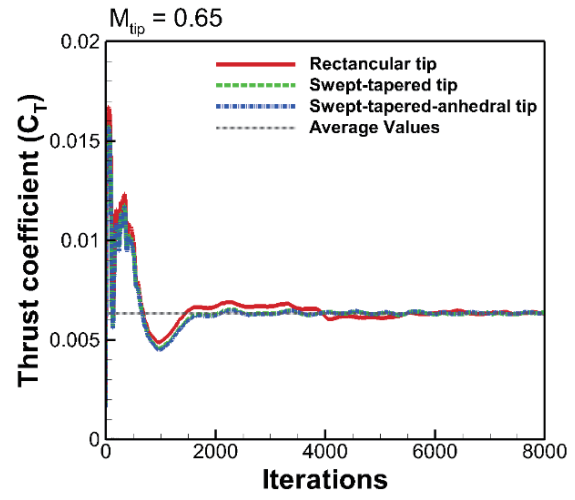

(a) Thrust coefficient vs Iterations

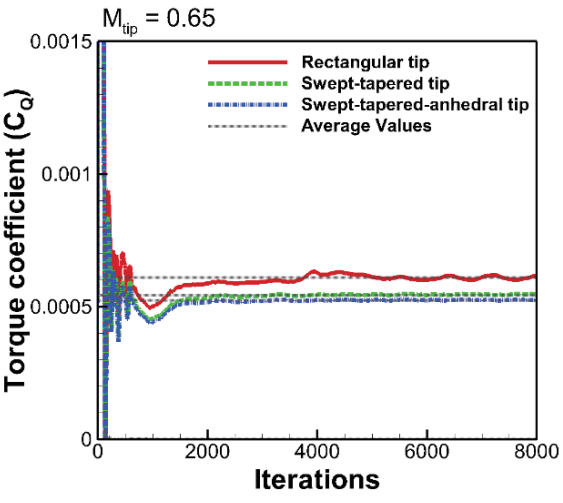

(b) Torque coefficient vs Iterations

Fig. 14. Convergence history of the thrust and torque coefficient at a specified thrust value of $C T / \sigma=0.09$ for the three blade tip shapes.

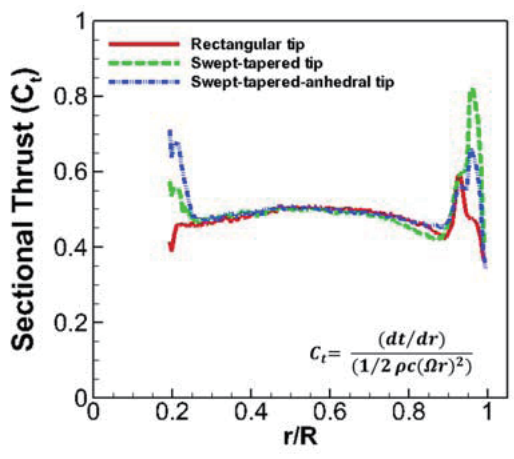

(a) Sectional thrust coefficient

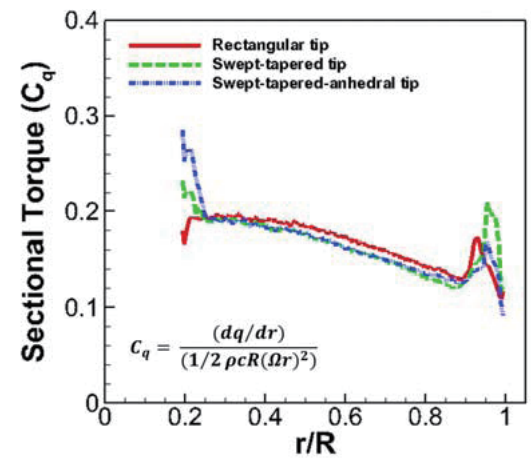

(b) Sectional torque coefficient

Fig. 15. Blade sectional thrust and torque distributions along the span at a specified thrust value of $C T / \sigma=0.09$ for the three blade tip shapes

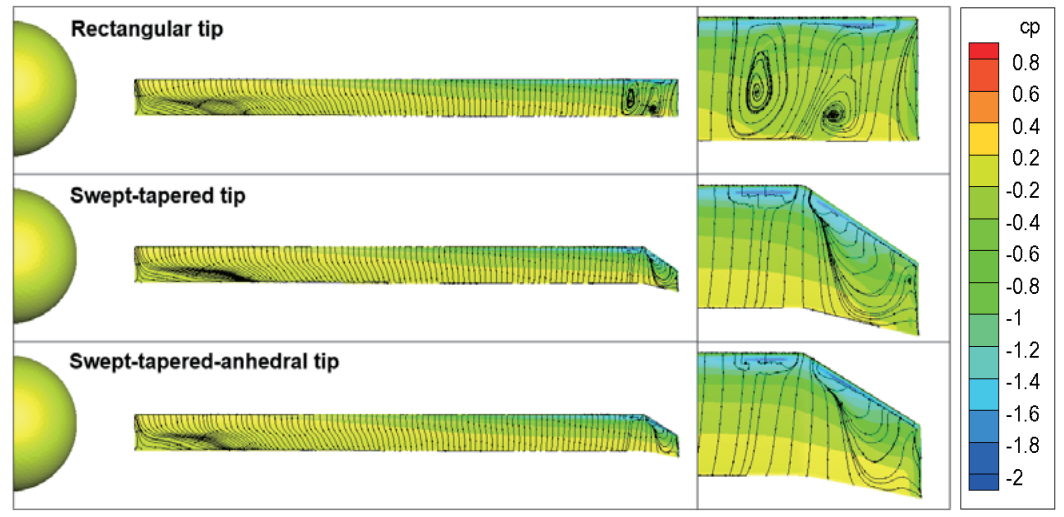

Fig. 16. Pressure coefficient contours and streamline patterns of the upper surface of the blade for three different tip shapes at a specified thrust value of $C_{T} / \sigma=0.09$ 
results are summarized in Table 1 . The converged collective pitch angle was revealed to be the highest for the rectangular tip, and the lowest for the swept-tapered-anhedral tip. The corresponding torque is the highest also for the rectangular tip and is the lowest for the swept-tapered-anhedral tip. Accordingly, the swept-tapered-anhedral tip exhibits the highest figure of merit.

The blade sectional thrust and torque coefficient distributions along the span are presented for the three blade tip shapes, as seen in Fig. 15. Both the sectional thrust loading and the torque are shown to have significantly increased at the tip due to the passage of the tip vortex from the proceeding blades. The peak values of those loadings are the lowest for the rectangular tip, partially due to the larger local blade chord length and the higher dynamic pressure at the tip.

The pressure contours and the streamline patterns are presented on the upper surface of the rotor blade in Fig. 16 for the three different tip shapes at a specified thrust value of $\mathrm{C}_{\mathrm{T}} / \sigma=0.09$. For the rectangular blade, a fairly strong flow separation can be observed at the tip, which induces low thrust loading and high torque at the tip, as shown in Fig. 15. Meanwhile, the leading-edge vortex is observed along the swept tip on the other two blades. The separated vortex for the swept-tapered-anhedral tip is slightly weaker than the swept-tapered tip as a result of the tip droop.

In Fig. 17, the pressure coefficient distribution along the chord at twelve selected radial stations is compared between the three different tip shapes at a specified thrust value of $\mathrm{C}_{\mathrm{T}}$ / $\sigma=0.09$. The inboard sections with an $\mathrm{r} / \mathrm{R}$ less than 0.8 reveal pressure distributions for all three blades that are almost identical to each other. However, at the outboard stations, a relatively large deviation is observed between the different tip shapes. Between an r/R of 0.8 and 0.925 , the difference appears mostly between the rectangular blade and the other two, mainly due to the separated flow pattern shown in Fig. 16. For further outboard stations, the effect of the leading-edge vortex for the two swept tips is clearly visible on the pressure distribution when compared to that of the rectangular blade. The strength of the leading-edge vortex is less for the blade with an anhedral tip than for the one without, as shown in Fig. 16.

In Fig. 18, the wake structures and the vorticity contours are compared between the three blade tip shapes at a specified thrust value of $\mathrm{C}_{\mathrm{T}} / \sigma=0.09$. The wake structures are represented by the iso- $\lambda 2$ surface colored by the vorticity magnitude. Vorticity contours are shown on the vertical plane along the reference blade. The overall wake structures are well resolved for all three cases by using the present flow solver, at least in the near wake region. The predicted wake structures are observed to be similar to each other for all three blade configurations. However, the figure clearly indicates that in the case of the rectangular tip, the first-passage vortex from the proceeding blade impinges on the following blade, while the tip vortex passes slightly underneath the lower surface of the blade for the other two blade configurations with swept tips. In the case of the anhedral tip, a secondary vortex is generated from the inboard, where the blade is kinked with a leading-edge sweep. The positions of the first passage vortex for each blade configuration are summarized in Table 2. The miss distance (d) is the first passage vortex distance from the leading edge.

In Fig. 19, the predicted tip vortex trajectories are compared for the three blade tip shapes in terms of the vortex age. The trajectories are presented for both the radial and the vertical positions for an azimuth angle from $0^{\circ}$ to $540^{\circ}$. In the figure, the initial reference vertical position was set at the trailing edge of the blade tip for each blade in a manner such that the vertical displacement can be consistently measured regardless of the blade tip shape. The radial contraction and the vertical convection distance are all similar to each other, at least for the near wake region. However, as the wake further develops, the tip vortex of the rectangular blade remains better resolved as that further downstream. In the case of the other two blades, the interaction between the layers of the tip vortices occurs at a relatively early stage, as shown in Fig. 18.

In Fig. 20, the development of the core radius and the circulation strength history of the tip vortex are presented in terms of the wake age. The tip-vortex core size can be determined by measuring the distance between the low and high peaks of the sectional circumferential velocity on the cutting plane of the tip vortex at each azimuthal position. As a result of the interaction between the tip vortices, the estimated core size shows a quite scattered behavior but gradually increases as the tip vortex travels further downstream. The circulation strength progressively decreased as a result of the dissipation mechanism for both the physical and numerical aspects.

Table 2. Comparison of the first passage vortex position between the three different blade tip shapes

\begin{tabular}{cccc}
\hline & Rectangular tip & Swept-tapered tip & Swept-tapered-anhedral tip \\
\hline Radial position $(\mathrm{r} / \mathrm{R})$ & 0.9209 & 0.9146 & 0.9170 \\
Vertical position $(-\mathrm{z} / \mathrm{R})$ & 0.0087 & 0.0201 & 0.0045 \\
Miss distance $(\mathrm{d} / \mathrm{R})$ & 0.0108 & 0.0221 & 0.0222 \\
\hline
\end{tabular}




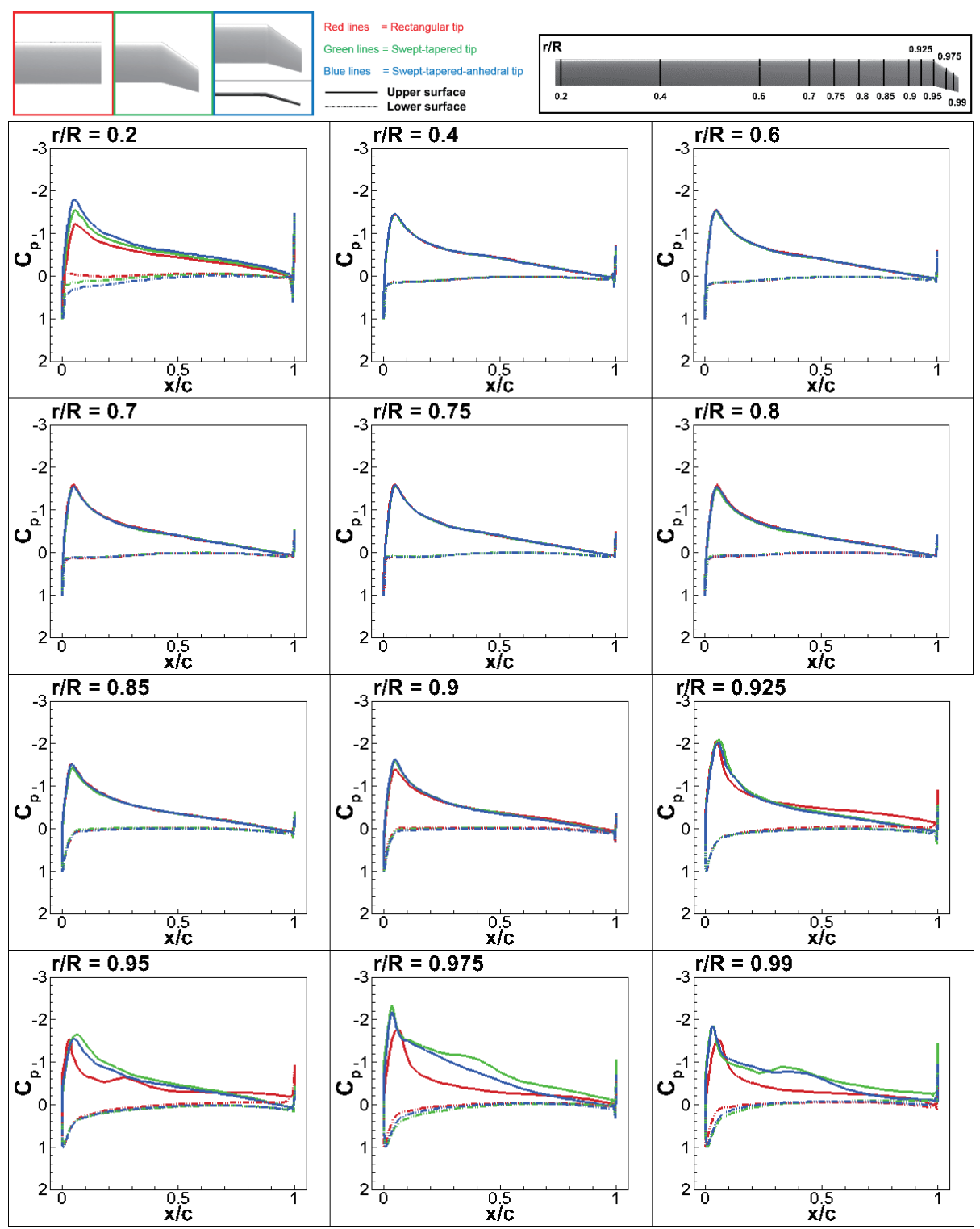

Fig. 17. Comparison of the chordwise surface pressure distributions at twelve radial stations between three different tip shapes at a specified thrust value of $C_{T} / \sigma=0.09$

\section{Conclusion}

In the present study, an unstructured mixed mesh flow solver was developed to simulate helicopter rotors in hover. The mixed mesh methodology involves a tetrahedral/ prismatic mesh in the near-body region around the rotor blades and at the center body and a Cartesian mesh in the off-body region. An overset mesh technique was adopted to communicate the flow variables between the two mesh regions. In the off-body region, the 7th-order WENO scheme was applied to improve the accuracy of the flow solution. The Spalart-Allmaras one-equation turbulence model was adopted to estimate the turbulent eddy viscosity.

The present flow solver was applied to an experimental S-76 helicopter rotor in hovering flight conditions. Computations were carried out for rotor blades operating with three different tip Mach numbers. Also, the effect of the blade tip shapes was investigated for rectangular, swept-taper, and swept-taper-anhedral shapes. Additional computations were also made for the three blade configurations to estimate the collective pitch angle of the trim blade for a specified rotor thrust setting. The simulations were carried out for various blade collective pitch angles, and the aerodynamic performance parameters and the flow 

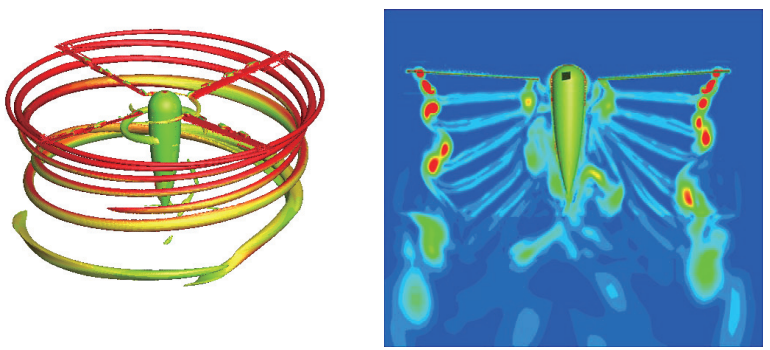

(a) Rectangular tip
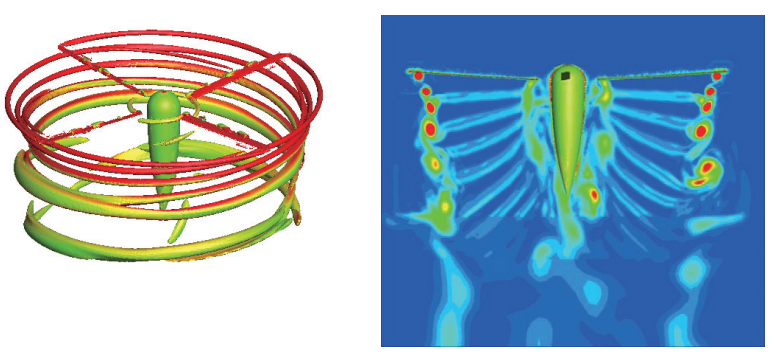

(b) Swept-tapered tip
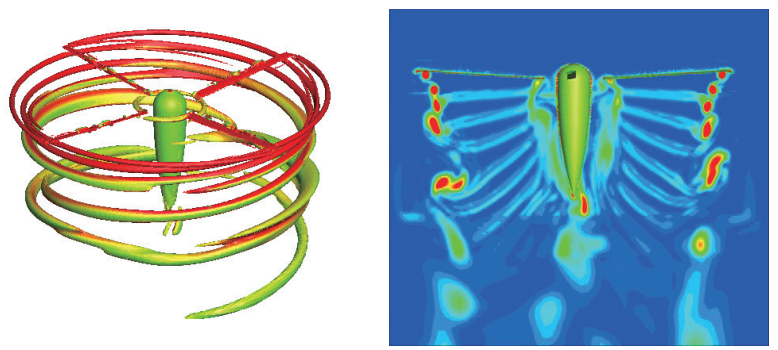

(c) Swept-tapered-anhedral tip

Fig. 18. Comparison of the wake structures and vorticity contours between three different tip shapes at specified thrust values of $C_{T} / \sigma=0.09$

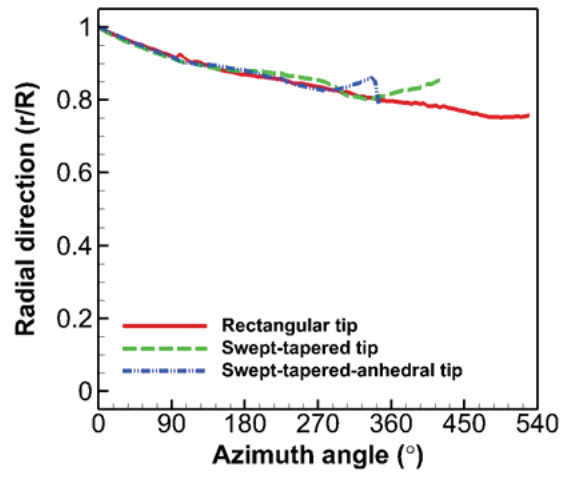

(a) Radial position

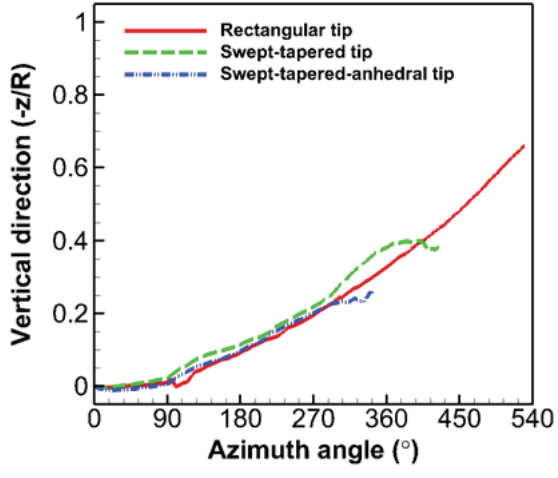

(b) Vertical position

Fig. 19. Comparison of the tip vortex trajectories between three different tip shapes at a specified thrust value of $C_{T} / \sigma=0.09$.

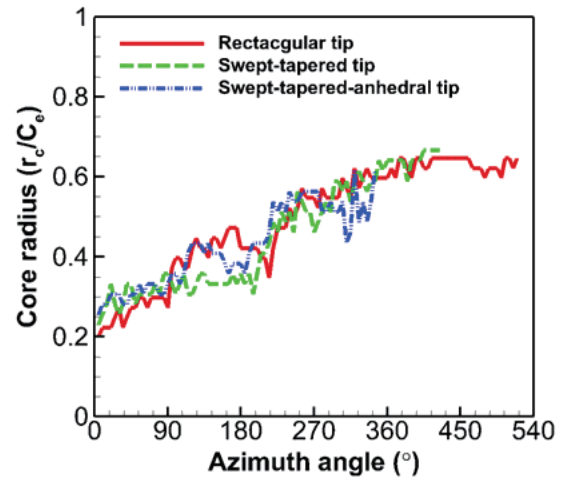

(a) Tip-vortex core size

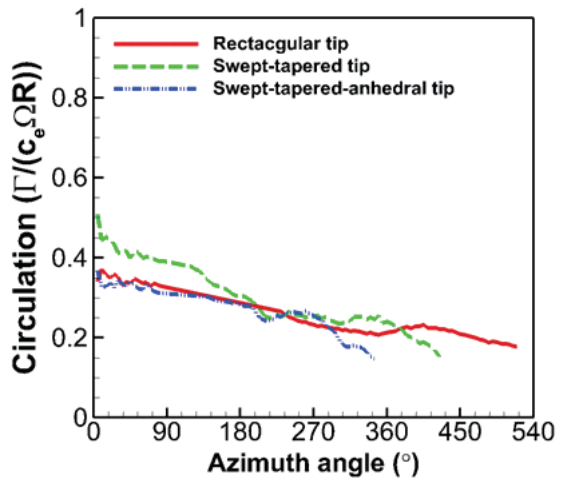

(b) Tip-vortex circulation strength

Fig. 20. Comparison of the tip-vortex core size and circulation strength between the three tip shapes at a specified thrust value of $C_{T} / \sigma=0.09$. 
behaviors were analyzed. The effect of the blade tip Mach number on the aerodynamic performance of hovering rotors was observed to become more significant at high collective pitch angles. The blade tip shape also has a significant effect on the blade tip aerodynamic loadings and the local flow behaviors. The present flow solver based on an unstructured mixed mesh topology coupled with high-order spatial accuracy was demonstrated to be capable of simulating the aerodynamic performance of a helicopter rotor in hovering flight conditions.

\section{Acknowledgement}

This research was supported by the Climate Change Research Hub of KAIST (Grant No. N01150026).This work was also supported with supercomputing resources, including technical support, by the National Institute of Supercomputing and Network/Korea Institute of Science and Technology Information (KSC-2014-C30-38).

\section{References}

[1] Weller, W. H., Experimental Investigation of Effects of Blade Tip Geometry on Loads and Performance for an Articulated Rotor System, NASA-TP-1303, 1979.

[2] Stroub, R. H., Rabbott, J. P. and Niebanck, C. F., "Rotor Blade Tip Shape Effects on Performance and Control Loads from Full-Scale Wind Tunnel Testing", Journal of the American Helicopter Society, Vol. 24, No. 4, 1979, pp. 28-35.

[3] Jepson, D., Moffitt, R., Hilzinger, K. and Bissell J., Analysis and Correlation of Test Data from an Advanced Technology Rotor System, NASA-CR-3714 c.1, 1980.

[4] Kim, K. C. and Chopar, I., "Aeroelastic Analysis of Swept, Anhedral, and Tapered Tip Rotor Blades", Journal of the American Helicopter Society, Vol. 37, No. 1, 1991, pp. 15-30.

[5] Yen, J. G., "Effects of Blade Tip Shape on Dynamics, Cost, Weight, Aerodynamic Performance, and Aeroelastic Response", Journal of the American Helicopter Society, Vol. 39, No. 4, 1994, pp. 37-45.

[6] Brocklehurst, A. and Barakos, G. N., "A Review of Helicopter Rotor Blade Tip Shapes", Progress in Aerospace Sciences, Vol. 56, 2013, pp. 35-74.

[7] Leishman, J. G., Principles of Helicopter Aerodynamics, Cambridge University Press, New York, 2006.

[8] Le Pape, A. and Beaumier, P., "Numerical Optimization of Helicopter Rotor Aerodynamic Performance in Hover", Aerospace Science and Technology, Vol. 9, No. 3, 2005, pp.
191-201.

[9] Aiken, E. W., Ormiston, R. A. and Young, L. A., "Future Directions in Rotorcraft Technology at Ames Research Center", $56^{\text {th }}$ annual forum of the American helicopter Society, Virginia Beach, VA, 2000.

[10] Brocklehurst, A., Steijl, R. and Barakos, G., "Using CFD to Understand and Evaluate Tail Rotor Blade Designs", AHS aeromechanics specialists' Conference, San Francisco, CA, 2008

[11] Jung, M. K. and Kwon, O. J., "Development of a 2-D Flow Solver on Unstructured and Adaptive Cartesian Meshes", Journal of Mechanical Science and Technology, Vol. 26, No. 12, 2012, pp. 3989-3997.

[12] Toro, E. F., Riemann Solvers and Numerical Methods for Fluid Dynamics: A Practical Introduction, SpringerVerlag, 1999.

[13] Shu, C. W. and Osher, S, "Efficient Implementation of Essentially Non-Oscillatory Shock-Capturing Schemes", Journal of Computational Physics, Vol. 77, No. 2, 1988, pp. 439-471.

[14] Shu, C. W. and Osher, S, "Efficient Implementation of Weighted ENO Schemes," Journal of Computational Physics, Vol. 126, No. 1, 1996, pp. 202-228.

[15] Visbal, M. R., and Gaitonde, D. V., "On the Use of Higher-Order Finite-Difference Schemes on Curvilinear and Deforming Meshes", Journal of Computational Physics, Vol. 181, No. 1, 2002, pp. 155-185.

[16] Barth, T. and Frederickson, P. O., "Higher Order Solution of the Euler Equations on Unstructured Grids Using Quadratic Reconstruction", 28 ${ }^{\text {th }}$ AIAA Aerospace Sciences Meeting, AIAA paper 90-0013, 1990.

[17] Buning, P. G. and Nichols, R. H., OVERFLOW User's Manual, NASA Langley Research Center, 2003.

[18] Wissink, A. M, Sitaraman, J., Sankaran, V., Mavriplis, D. J. and Pulliam, T. H., "A Multi-Code Python-Based Infrastructure for Overset CFD with Adaptive Cartesian Grids", $46^{\text {th }}$ AIAA Aerospace Science Meeting, AIAA 2008-927, 2008.

[19] Mavriplis, D. J. and Venkatakrishnan, V., "A Unified Multigrid Solver for the Navier-Stokes Equations on Mixed Element Meshes", International Journal for Computational Fluid Dynamics, Vol. 8, 1997, pp. 247-263.

[20] Hornung, R. D., Wissink, A. M. and Kohn, S. R., "Managing Complex Data and Geometry in Parallel Structured AMR Applications", Engineering with Computers, Vol. 22, No. 3-4, 2006, pp. 181-195.

[21] Pulliam, T. H., "Euler and Thin-Layer Navier-Stokes Codes: ARC2D and ARC3D", Computational Fluid Dynamics User Workshop, 1984.

[22] Schluter, J. U., Wu, X., Weide, E., Hahn, S., Alonso, J. 
J., and Pitsch, H., "Multi-Code Simulations: A Generalized Coupling Approach", $17^{\text {th }}$ AIAA Computational Fluid Dynamics Conference, AIAA 2005-4997, 2005.

[23] Balch, D. T. and Lombardi, J., Experimental Study of Main Rotor Tip Geometry and Tail Rotor Interactions in Hover Vol I - TEXT and FIGURES, NASA CR-177336-Vol-1, 1985.

[24] Balch, D. T. and Lombardi, J., Experimental Study of Main Rotor Tip Geometry and Tail Rotor Interactions in Hover Vol II - RUN LOG and TABULATED DATA, NASA CR177336-Vol-2, 1985.

[25] Shur, M. L., Strelets, M. K., Travin, A. K., and Spalart, P. R., "Turbulence Modeling in Rotating and Curved Channels: Assessing the Spalart-Shur Correction", AIAA Journal, Vol. 38, No. 5, 2000, pp. 784-792.

[26] Venkatakrishnan, V., "Convergence to Steady State Solutions of the Euler Equations on Unstructured Grids with Limiters", Journal of Computational Physics, Vol. 118, No. 1,
1995, pp. 120-130.

[27] Aftosmis, M. J., Solution Adaptive Cartesian Grid Methods for Aerodynamic Flows with Complex Geometries, Von Karman Institute for Fluid Dynamics, Lecture Series, 1997.

[28] Liu, X. D., Osher, S. and Chan, T., "Weighted Essentially Non-oscillatory Schemes", Journal of Computational Physics, Vol. 115, 1994, pp. 200-212.

[29] Jiang, G. S. and Shu, C. W., "Efficient Implementation of Weighted ENO Schemes", Journal of Computational Physics, Vol. 126, 1996, pp. 202-228.

[30] Shen, Y. Q. and Zha G. C., "A Robust Seventh-order WENO Scheme and Its Applications", AIAA paper 2008-0757, 2008.

[31] Karypis, G. and Kumar, V., "Multilevel k-way Partitioning Schemes for Irregular Graphs,, Journal of Parallel and Distributed Computing, Vol. 48, No. 1, 1998, pp. 96-129. 